Article

\title{
A Novel Delay-Dependent Asymptotic Stability Conditions for Differential and Riemann-Liouville Fractional Differential Neutral Systems with Constant Delays and Nonlinear Perturbation
}

\author{
Watcharin Chartbupapan ${ }^{1}(\mathbb{D})$, Ovidiu Bagdasar ${ }^{2}(\mathbb{D})$ and Kanit Mukdasai ${ }^{1, *(D)}$ \\ 1 Department of Mathematics, Faculty of Science, Khon Kaen University, khon Kaen 40002, Thailand; \\ chartbupapan@gmail.com \\ 2 Department of Electronics, Computing and Mathematics, University of Derby, Derby DE22 1GB, UK; \\ o.bagdasar@derby.ac.uk \\ * Correspondence: kanit@kku.ac.th
}

Received: 28 November 2019; Accepted: 23 December 2019; Published: 3 January 2020

\begin{abstract}
The novel delay-dependent asymptotic stability of a differential and Riemann-Liouville fractional differential neutral system with constant delays and nonlinear perturbation is studied. We describe the new asymptotic stability criterion in the form of linear matrix inequalities (LMIs), using the application of zero equations, model transformation and other inequalities. Then we show the new delay-dependent asymptotic stability criterion of a differential and Riemann-Liouville fractional differential neutral system with constant delays. Furthermore, we not only present the improved delay-dependent asymptotic stability criterion of a differential and Riemann-Liouville fractional differential neutral system with single constant delay but also the new delay-dependent asymptotic stability criterion of a differential and Riemann-Liouville fractional differential neutral equation with constant delays. Numerical examples are exploited to represent the improvement and capability of results over another research as compared with the least upper bounds of delay and nonlinear perturbation.
\end{abstract}

Keywords: asymptotic stability; differential and riemann-liouville fractional differential neutral systems; linear matrix inequality

\section{Introduction}

Differential systems, or more generally functional differential systems, have been studied rather extensively for at least 200 years and are used as models to describe transportation systems, communication networks, teleportation systems, physical systems and biological systems, and so forth. Parts of fractional-order systems have not received much attention by reason of absence of appropriate utilization circumstances over the past 300 years. However, during the last 10 years fractional-order systems have been widely investigated as they have the qualification to explain various phenomena more precisely in many fields, for example, biological models, material science, finance, cardiac tissues, quantum mechanics, viscoelastic systems, medicine and fluid mechanics [1-8]. Caputo fractional differential systems have been studied in many types of stability such as uniform stability [9], Mittag-Leffler stability [10-13], Ulam stability [14], finite time stability [15,16] and asymptotic stability $[17,18]$. Nevertheless, the stability of Riemann-Liouville fractional differential systems is seldom considered, see References $[19,20]$.

The neutral systems with time delays have already been applied in many fields, such as heartbeat, memorization, locomotion, mastication and respiration, see References [21-24]. Accordingly, the issue 
of stability analysis for differential and Riemann-Liouville fractional differential neutral systems has attracted researchers. The asymptotic stability criteria for certain neutral differential equations (CNDE) with constant delays have been discussed in References [25-29] by applying Lyapunov-Krasovskii functional and several model transformations. In References [30-33], the researchers considered the exponential stability problem for CNDE with time-varying delays by several methods. In Reference [30], the results were established without the use of the bounding technique and the model transformation method, while researchers have studied it by using radially unboundedness, the Lyapunov-Krasovskii functional approach and the model transformation method in Reference [32]. Moreover, in Reference [34] Li et al. presented the asymptotic stability conditions for fractional neutral systems in the form of matrix measure and matrix norm of the system matrices. However, the criteria, drafted in the form of matrix norm, are more conservative, while Liu et al. used the Lyapunov direct method to establish the asymptotic stability criteria of Riemann-Liouville fractional neutral systems in the form of LMIs [35].

This paper is involved with the analysis problem for the asymptotic stability of differential and Riemann-Liouville fractional differential neutral systems with constant delays and nonlinear perturbation by applying a zero equation, model transformation and other inequalities. The novel asymptotic stability condition is instituted in the form of LMIs. Then we show the new delay-dependent asymptotic stability criterion of differential and Riemann-Liouville fractional differential neutral systems with constant delays. In addition, the improved delay-dependent asymptotic stability criterion of differential and Riemann-Liouville fractional differential neutral systems with single constant delay and the new delay-dependent asymptotic stability criterion of differential and Riemann-Liouville fractional differential neutral equations with constant delays are established. Numerical examples represent the capability of our results as compared with other research.

\section{Problem Formulation and Preliminaries}

We introduce a differential and fractional differential neutral system with constant delays and nonlinear perturbation

$$
\begin{aligned}
t_{0} D_{t}^{q}[x(t)+C x(t-\tau)] & =-A x(t)+B x(t-\sigma)+f(x(t-\sigma)), \quad t>0, \\
x(t) & =\varrho(t), \quad t \in[-\kappa, 0],
\end{aligned}
$$

for $0<q \leq 1$, the state vector $x(t) \in \mathbb{R}^{n}, A, B, C$ are symmetric positive definite matrices with $\|C\|<1$, $\tau, \sigma$ are positive real constants and $\varrho \in C\left([-\kappa, 0] ; \mathbb{R}^{n}\right)$ with $\kappa=\max \{\tau, \sigma\}$.

The uncertainty $f($.$) represents the nonlinear parameter perturbation satisfying$

$$
\begin{aligned}
f^{T}(x(t)) f(x(t)) & \leq \delta^{2} x^{T}(t) x(t), \\
f^{T}(x(t-\sigma)) f(x(t-\sigma)) & \leq \eta^{2} x^{T}(t-\sigma) x(t-\sigma),
\end{aligned}
$$

where $\delta, \eta$ are given constants.

Next, the Riemann-Liouville fractional integral and derivative [36] are defined as, respectively

$$
\begin{aligned}
t_{0} D_{t}^{-q} x(t) & =\frac{1}{\Gamma(q)} \int_{t_{0}}^{t}(t-s)^{q-1} x(s) d s, \quad(q>0), \\
t_{0} D_{t}^{q} x(t) & =\frac{1}{\Gamma(n-q)} \frac{d^{n}}{d t^{n}} \int_{t_{0}}^{t} \frac{x(s)}{(t-s)^{q+1-n}} d s, \quad(n-1 \leq q<n) .
\end{aligned}
$$

Lemma 1. [37] For $x(t) \in \mathbb{R}^{n}$ and $p>q>0$, then

$$
t_{0} D_{t}^{q}\left(t_{0} D_{t}^{-p} x(t)\right)={ }_{t_{0}} D_{t}^{q-p} x(t) .
$$


Lemma 2. [17] For a vector of differentiable function $x(t) \in \mathbb{R}^{n}$, positive semi-definite matrix $K \in \mathbb{R}^{n \times n}$ and $0<q<1$, then

$$
\frac{1}{2} t_{0} D_{t}^{q}\left(x^{T}(t) K x(t)\right) \leq x^{T}(t) K_{t_{0}} D_{t}^{q} x(t)
$$

for all $t \geq t_{0}$.

\section{Main Results}

Consider the asymptotic stability for system (1) with constant delays and nonlinear perturbation. We define a new variable

$$
\Psi(t)=x(t)+C x(t-\tau)
$$

Rewrite the Equation (1) in the following equation

$$
{ }_{t_{0}} D_{t}^{q} \Psi(t)=-A x(t)+B x(t-\sigma)+f(x(t-\sigma))
$$

Theorem 1. Let $\delta$ and $\eta$ be positive scalars, if there are any appropriate dimensions matrices $Q_{j}(j=1,2,3)$ and symmetric positive definite matrices $K_{i}(i=1,2,3,4,5)$ such that satisfy

$$
\sum=\left[\begin{array}{ccccc}
-Q_{1}-Q_{1}^{T} & \Omega_{(1,2)} & Q_{1} C-Q_{3}^{T} & K_{1} & K_{1} B \\
* & \Omega_{(2,2)} & Q_{2} C+Q_{3}^{T} & 0 & 0 \\
* & * & \Omega_{(3,3)} & 0 & 0 \\
* & * & * & -K_{5}-\sigma I & 0 \\
* & * & * & * & -K_{3}+\sigma \eta^{2} I
\end{array}\right]<0
$$

where

$\Omega_{(1,2)}=-K_{1} A+Q_{1}-Q_{2}^{T}$,

$\Omega_{(2,2)}=Q_{2}+Q_{2}^{T}+K_{2}+K_{3}+\tau K_{4}+\delta^{2} K_{5}$,

$\Omega_{(3,3)}=Q_{3} C+C^{T} Q_{3}^{T}-K_{2}$.

Then the system (1) is asymptotically stable.

Proof of Theorem 1. For symmetric positive definite matrices $K_{i}(i=1,2,3,4,5)$ and any appropriate dimensions matrices $Q_{j}(j=1,2,3)$. Consider the Lyapunov-Krasovskii functional

$$
V(t)=\sum_{i=1}^{2} V_{i}(t),
$$

for

$$
\begin{aligned}
V_{1}(t)= & t_{0} D_{t}^{q-1} \Psi^{T}(t) K_{1} \Psi(t) \\
V_{2}(t)= & \int_{t-\tau}^{t} x^{T}(s) K_{2} x(s) d s+\int_{t-\sigma}^{t} x^{T}(s) K_{3} x(s) d s \\
& +\int_{t-\tau}^{t}(\tau-t+s) x^{T}(s) K_{4} x(s) d s \\
& +\int_{t-\sigma}^{t} f^{T}(x(s)) K_{5} f(x(s)) d s .
\end{aligned}
$$

Computing the differential of $V(t)$ on the solution of system (1) 


$$
\dot{V}(t)=\sum_{i=1}^{2} \dot{V}_{i}(t) .
$$

The differential of $V_{1}(t)$ is computed by Lemma 2

$$
\begin{aligned}
\dot{V}_{1}(t)= & t_{0} D_{t}^{q} \Psi^{T}(t) K_{1} \Psi(t) \\
\leq & 2 \Psi^{T}(t) K_{1}\left(t_{0} D_{t}^{q} \Psi(t)\right) \\
= & 2 \Psi^{T}(t) K_{1}[-A x(t)+B x(t-\sigma)+f(x(t-\sigma))] \\
& +2 \Psi^{T}(t) Q_{1}[-\Psi(t)+x(t)+C x(t-\tau)] \\
& +2 x^{T}(t) Q_{2}[-\Psi(t)+x(t)+C x(t-\tau)] \\
& +2 x^{T}(t-\tau) Q_{3}[-\Psi(t)+x(t)+C x(t-\tau)]
\end{aligned}
$$

Taking the differential of $V_{2}(t)$, we obtain

$$
\begin{aligned}
\dot{V}_{2}(t)= & x^{T}(t) K_{2} x(t)-x^{T}(t-\tau) K_{2} x(t-\tau) \\
& +x^{T}(t) K_{3} x(t)-x^{T}(t-\sigma) K_{3} x(t-\sigma) \\
& +\tau x^{T}(t) K_{4} x(t)-\int_{t-\tau}^{t} x^{T}(s) K_{4} x(s) \\
& +f^{T}(x(t)) K_{5} f(x(t))-f^{T}(x(t-\sigma)) K_{5} f(x(t-\sigma)) \\
\leq & x^{T}(t) K_{2} x(t)-x^{T}(t-\tau) K_{2} x(t-\tau) \\
& +x^{T}(t) K_{3} x(t)-x^{T}(t-\sigma) K_{3} x(t-\sigma) \\
& +\tau x^{T}(t) K_{4} x(t)+\delta^{2} x^{T}(t) K_{5} x(t) \\
& -f^{T}(x(t-\sigma)) K_{5} f(x(t-\sigma)) .
\end{aligned}
$$

Next, from (3), we obtain

$$
0 \leq \sigma \eta^{2} x^{T}(t-\sigma) x(t-\sigma)-\sigma f^{T}(x(t-\sigma)) f(x(t-\sigma)) .
$$

According to (13), (14) and (15), we can conclude that

$$
\dot{V}(t) \leq \xi^{T}(t) \sum \xi(t),
$$

where $\xi(t)=\operatorname{col}\left\{\Psi(t), x(t), x(t-\tau), f(x(t-\sigma)), x^{T}(t-\sigma)\right\}$.

Since linear matrix inequality (10) holds, then the system (1) is asymptotic stability.

Next, we consider system (1) with $f(x(t-\sigma))=0$,

$$
\begin{aligned}
t_{0} D_{t}^{q}[x(t)+C x(t-\tau)] & =-A x(t)+B x(t-\sigma) \quad t>0, \\
x(t) & =\varrho(t), \quad t \in[-\kappa, 0],
\end{aligned}
$$

for $0<q \leq 1$, the state vector $x(t) \in \mathbb{R}^{n}, A, B, C$ are symmetric positive definite matrices with $\|C\|<1$, $\tau, \sigma$ are positive real constants and $\varrho \in C\left([-\kappa, 0] ; \mathbb{R}^{n}\right)$ with $\kappa=\max \{\tau, \sigma\}$.

Corollary 1. If there are any appropriate dimensions matrices $Q_{j}(j=1,2,3)$ and symmetric positive definite matrices $K_{i}(i=1,2,3,4)$ such that satisfy

$$
\left[\begin{array}{cccc}
-Q_{1}-Q_{1}^{T} & -K_{1} A+Q_{1}-Q_{2}^{T} & Q_{1} C-Q_{3}^{T} & K_{1} B \\
* & Q_{2}+Q_{2}^{T}+K_{2}+K_{3}+\tau K_{4} & Q_{2} C+Q_{3}^{T} & 0 \\
* & * & Q_{3} C+C^{T} Q_{3}^{T}-K_{2} & 0 \\
* & * & * & -K_{3}
\end{array}\right]<0 .
$$


Then the system (17) is asymptotically stable.

Proof of Corollary 1. For symmetric positive definite matrices $K_{i}(i=1,2,3,4)$ and any appropriate dimensions matrices $Q_{j}(j=1,2,3)$. Consider the Lyapunov-Krasovskii functional

$$
V(t)=\sum_{i=1}^{2} V_{i}(t)
$$

for

$$
\begin{aligned}
V_{1}(t)= & t_{0} D_{t}^{q-1} \Psi^{T}(t) K_{1} \Psi(t) \\
V_{2}(t)= & \int_{t-\tau}^{t} x^{T}(s) K_{2} x(s) d s+\int_{t-\sigma}^{t} x^{T}(s) K_{3} x(s) d s \\
& +\int_{t-\tau}^{t}(\tau-t+s) x^{T}(s) K_{4} x(s) d s .
\end{aligned}
$$

According to Theorem 1, we present the asymptotic stability criterion (18) of system (17).

Next, we consider system (1) with $f(x(t-\sigma))=0$ and $\sigma=\tau$,

$$
\begin{aligned}
t_{0} D_{t}^{q}[x(t)+C x(t-\tau)] & =-A x(t)+B x(t-\tau) \quad t>0, \\
x(t) & =\varrho(t), \quad t \in[-\tau, 0],
\end{aligned}
$$

for $0<q \leq 1$, the state vector $x(t) \in \mathbb{R}^{n}, A, B, C$ are symmetric positive definite matrices with $\|C\|<1$, $\tau$ is positive real constants and $\varrho \in C\left([-\tau, 0] ; \mathbb{R}^{n}\right)$.

Corollary 2. If there are any appropriate dimensions matrices $Q_{j}(j=1,2,3)$ and symmetric positive definite matrices $K_{i}(i=1,2,3)$ such that satisfy

$$
\left[\begin{array}{ccc}
-Q_{1}-Q_{1}^{T} & -K_{1} A+Q_{1}-Q_{2}^{T} & Q_{1} C-Q_{3}^{T}+K_{1} B \\
* & Q_{2}+Q_{2}^{T}+K_{2}+\tau K_{3} & Q_{2} C+Q_{3}^{T} \\
* & * & Q_{3} C+C^{T} Q_{3}^{T}-K_{2}
\end{array}\right]<0
$$

Then the Equation (20) is asymptotically stable.

Proof of Corollary 2. For symmetric positive definite matrices $K_{i}(i=1,2,3)$ and any appropriate dimensions matrices $Q_{j}(j=1,2,3)$. Consider the Lyapunov-Krasovskii functional

$$
V(t)=\sum_{i=1}^{2} V_{i}(t),
$$

for

$$
\begin{aligned}
V_{1}(t)= & t_{0} D_{t}^{q-1} \Psi^{T}(t) K_{1} \Psi(t) \\
V_{2}(t)= & \int_{t-\tau}^{t} x^{T}(s) K_{2} x(s) d s \\
& +\int_{t-\tau}^{t}(\tau-t+s) x^{T}(s) K_{3} x(s) d s .
\end{aligned}
$$

According to Theorem 1, we present the asymptotic stability criterion (21) of system (20). 


\section{Application}

$$
\begin{aligned}
t_{0} D_{t}^{q}[x(t)+p x(t-\tau)] & =-a x(t)+b \tanh x(t-\sigma) \quad t>0 \\
x(t) & =\varrho(t), \quad t \in[-\kappa, 0]
\end{aligned}
$$

for $0<q \leq 1$, the state vector $x(t) \in \mathbb{R}, a, b, p$ are real constants with $|p|<1, \tau, \sigma$ are positive real constants $\varrho \in C([-\kappa, 0] ; \mathbb{R})$ with $\kappa=\max \{\tau, \sigma\}$.

Corollary 3. If there are positive real constants $k_{i}(i=1,2,3,4,5)$ and real constants $q_{j}(j=1,2,3)$ such that satisfy

$$
\left[\begin{array}{ccccc}
-2 q_{1} & -k_{1} a+q_{1}-q_{2} & q_{1} p-q_{3} & k_{1} b & 0 \\
* & 2 q_{2}+k_{2}+k_{3}+k_{4} \tau+k_{5} & q_{2} p+q_{3} & 0 & 0 \\
* & * & 2 q_{3} p-k_{2} & 0 & 0 \\
* & * & * & -k_{5}-\sigma & 0 \\
* & * & * & * & -k_{3}+\sigma
\end{array}\right]<0
$$

Then the Equation (24) is asymptotically stable.

Proof of Corollary 3. For positive real constants $k_{i}(i=1,2,3,4,5)$ and real constants $q_{j}(j=1,2,3)$. Consider the Lyapunov-Krasovskii functional

$$
V(t)=\sum_{i=1}^{2} V_{i}(t)
$$

for

$$
\begin{aligned}
V_{1}(t)= & k_{1 t_{0}} D_{t}^{q-1} \Psi^{2}(t) \\
V_{2}(t)= & k_{2} \int_{t-\tau}^{t} x^{2}(s) d s+k_{3} \int_{t-\sigma}^{t} x^{2}(s) d s \\
& +k_{4} \int_{t-\tau}^{t}(\tau-t+s) x^{2}(s) d s+k_{5} \int_{t-\sigma}^{t} \tanh x^{2}(s) d s .
\end{aligned}
$$

According to Theorem 1, we present the asymptotic stability criterion (25) of system (3).

\section{Numerical Examples}

Example 1. The fractional neutral system :

$$
{ }_{t_{0}} D_{t}^{q}[x(t)+C x(t-0.5)]=-A x(t)+B x(t-\sigma)+f(x(t-\sigma)) .
$$

Solving the LMI (10) when $A=\left[\begin{array}{cc}1.45 & 0 \\ 0 & 1.45\end{array}\right], B=\left[\begin{array}{cc}0 & 0.4 \\ 0.4 & 0\end{array}\right], C=\left[\begin{array}{cc}-0.1 & 0 \\ 0 & -0.1\end{array}\right]$, we have a set of parameters that ensures asymptotic stability of system (27) which $\eta=5 \times 10^{3}, \delta=1$ and $\sigma=0.5$ as follows:

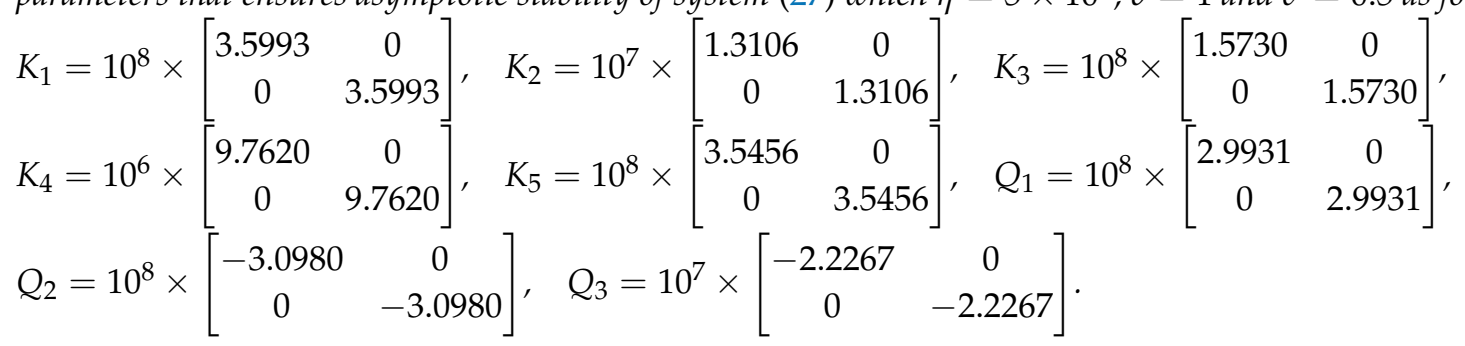


Moreover, the least upper bound of the parameter $\sigma$ that ensures the asymptotic stability of system (27) is 1.3227 when $\eta=5 \times 10^{3}$ and $\delta=1$. Table 1 represents the least upper bound $\sigma$ of this example for various values of $\eta, \delta$.

Table 1. The least upper bound of $\sigma$ for Example 1.

\begin{tabular}{cccc}
\hline & $\boldsymbol{\eta}=\mathbf{5} \times \mathbf{1 0}^{\mathbf{3}}$ & $\boldsymbol{\eta}=\mathbf{6} \times \mathbf{1 0}^{\mathbf{3}}$ & $\boldsymbol{\eta}=\mathbf{7} \times \mathbf{1 0}^{\mathbf{3}}$ \\
\hline$\delta=0.8$ & 6.4920 & 4.5076 & 3.3117 \\
$\delta=0.9$ & 4.1166 & 2.8588 & 2.1003 \\
$\delta=1$ & 1.3227 & 0.9185 & 0.6748 \\
\hline
\end{tabular}

Example 2. The fractional neutral system:

$$
t_{0} D_{t}^{q}[x(t)+C x(t-\tau)]=-A x(t)+B x(t-1.2) .
$$

Solving the LMI (18) when $A=\left[\begin{array}{cc}1.45 & 0 \\ 0 & 1.45\end{array}\right], B=\left[\begin{array}{cc}0 & 0.4 \\ 0.4 & 0\end{array}\right], C=\left[\begin{array}{cc}-0.1 & 0 \\ 0 & -0.1\end{array}\right]$, we have a set of parameters that ensures asymptotic stability of system (28) which $\tau=0.6$ as follows:

$$
\begin{gathered}
K_{1}=\left[\begin{array}{cc}
44.0782 & 0 \\
0 & 44.0782
\end{array}\right], \quad K_{2}=\left[\begin{array}{cc}
32.9861 & 0 \\
0 & 32.9861
\end{array}\right], \quad K_{3}=\left[\begin{array}{cc}
32.6501 & 0 \\
0 & 32.6501
\end{array}\right], \\
K_{4}=\left[\begin{array}{cc}
31.8793 & 0 \\
0 & 31.8793
\end{array}\right], \quad Q_{1}=\left[\begin{array}{cc}
14.6090 & 0 \\
0 & 14.6090
\end{array}\right], \quad Q_{2}=\left[\begin{array}{cc}
-56.7801 & 0 \\
0 & -56.7801
\end{array}\right], \\
Q_{3}=\left[\begin{array}{cc}
-3.3600 & 0 \\
0 & -3.3600
\end{array}\right] .
\end{gathered}
$$

Moreover, the least upper bound of the parameter $\tau$ that ensures the asymptotic stability of system (28) is $3.7 \times 10^{22}$.

Example 3. The fractional neutral system :

$$
t_{0} D_{t}^{q}[x(t)+C x(t-\tau)]=-A x(t)+B x(t-\tau) .
$$

Solving the LMI (21) when $A=\left[\begin{array}{cc}3 & -1 \\ 0 & 1\end{array}\right], B=\left[\begin{array}{cc}0.2 & 0.1 \\ 0 & 0.1\end{array}\right], C=\left[\begin{array}{cc}0.1 & 0 \\ 0 & 0.2\end{array}\right]$, we obtain the least upper bound of the parameter $\tau$ that ensures the asymptotic stability is $2.86 \times 10^{24}$. By the criterion in [35], the least upper bound of the parameter $\tau$ is $2.99 \times 10^{21}$. This example represents our result is less conservative than these in [35].

Example 4. The differential equation, which is considered in [25,27,30-32]:

$$
\frac{d}{d t}[x(t)+0.35 x(t-0.5)]=-1.5 x(t)+b \tanh x(t-0.5) .
$$

By using linear matrix inequality (25), the comparison for the least upper bound $b$ that ensures asymptotic stability of Equation (30) are represented in Table 2. 
Table 2. The least upper bound of $b$ for Example 4 .

\begin{tabular}{cc}
\hline Deng et al. (2009) [25] & 0.889 \\
Nam and Phat (2009) [27] & 1.405 \\
Chen and Meng (2011) [31] & 1.346 \\
Chen (2012) [30] & 1.405 \\
Keadnarmol and Rojsiraphisal (2014) [32] & 1.405 \\
Corollary 3 & 1.4051 \\
\hline
\end{tabular}

Example 5. The differential equation in [27,30,31,38]:

$$
\frac{d}{d t}[x(t)+0.2 x(t-0.1)]=-0.6 x(t)+0.3 \tanh x(t-\sigma) .
$$

By using linear matrix inequality (25), the comparison for the least upper bound delay $\sigma$ that ensures asymptotic stability of Equation (31) are represented in Table 3.

Table 3. The least upper bound of $\sigma$ for Example 5.

\begin{tabular}{cc}
\hline Nam and Phat (2009) [27] & 2.32 \\
Rojsiraphisal and Niamsup (2010) [38] & 2.32 \\
Chen and Meng (2011) [31] & $10^{21}$ \\
Chen (2012) [30] & $1.34 \times 10^{21}$ \\
Corollary 3 & $6.21 \times 10^{8}$ \\
\hline
\end{tabular}

Example 6. The fractional neutral equation:

$$
t_{0} D_{t}^{q}[x(t)+p x(t-0.5)]=-a x(t)+b \tanh x(t-0.5) .
$$

Solving the LMI (25), we have a set of parameters that ensures asymptotic stability of Equation (32) which $a=0.75, b=0.3$ and $p=0.4$ as follows:

$k_{1}=3.1544, \quad k_{2}=1.0324, \quad k_{3}=1.0749, \quad k_{4}=0.7170, \quad k_{5}=0.7385, \quad q_{1}=0.7587, \quad q_{2}=-1.9721$, $q_{3}=0.4433$.

Furthermore, the least upper bound of $b$ that ensures the asymptotic stability of Equation (32) is 0.6873 with $a=0.75, p=0.4$. Table 4 represents the least upper bound $b$ of this example for various values of $a, p$.

Table 4. The least upper bound of $b$ for Example 6.

\begin{tabular}{lccccc}
\hline & $\boldsymbol{a}=\mathbf{0 . 2 5}$ & $\boldsymbol{a}=\mathbf{0 . 5}$ & $\boldsymbol{a}=\mathbf{0 . 7 5}$ & $\boldsymbol{a}=\mathbf{1}$ & $\boldsymbol{a}=\mathbf{1 . 2 5}$ \\
\hline$p=0.2$ & 0.2449 & 0.4898 & 0.7348 & 0.9797 & 1.2247 \\
$p=0.4$ & 0.2291 & 0.4582 & 0.6873 & 0.9165 & 1.1456 \\
$p=0.6$ & 0.2000 & 0.3999 & 0.5999 & 0.7999 & 0.9999 \\
$p=0.8$ & 0.1500 & 0.2999 & 0.4499 & 0.5999 & 0.7499 \\
\hline
\end{tabular}

\section{Conclusions}

The aim of this paper is a novel asymptotic stability analysis of differential and Riemann-Liouville fractional differential neutral systems with constant delays and nonlinear perturbation by applying zero equations, model transformation and other inequalities. The new asymptotic stability condition is given in the form of LMIs. Then we show the new delay-dependent asymptotic stability criterion of a differential and Riemann-Liouville fractional differential neutral system with constant delays. Furthermore, we propose the improved delay-dependent asymptotic stability criterion of differential and Riemann-Liouville fractional differential neutral systems with single constant delay and the new delay-dependent asymptotic stability criterion of differential and Riemann-Liouville fractional differential neutral equations with constant delays. Numerical examples illustrate the advantages and applicability of our results. 
Author Contributions: All authors claim to have contributed significantly and equally to this work. All authors have read and agreed to the published version of the manuscript.

Funding: This work is supported by Science Achievement Scholarship of Thailand (SAST), Research and Academic Affairs Promotion Fund, Faculty of Science, Khon Kaen University, Fiscal year 2020 and National Research Council of Thailand and Khon Kaen University, Thailand (6200069).

Acknowledgments: The authors thank the reviewers for their valuable comments and suggestions, which led to the improvement of the content of the paper.

Conflicts of Interest: The authors declare no conflict of interest.

\section{References}

1. Ahmad, B.; Alghanmi, M.; Alsaedi, A.; Agarwal, R.V. Nonlinear impulsive multi-order Caputo-Type generalized fractional differential equations with infinite delay. Mathematics 2019, 7, 1108. [CrossRef]

2. Khan, U.; Ellahi, R.; Khan, R.; Mohyud-Din, S.T. Extracting new solitary wave solutions of Benny-Luke equation and Phi-4 equation of fractional order by using $\left(\mathrm{G}^{\prime} / \mathrm{G}\right)$-expansion method. Opt. Quant. Electron. 2017, 49, 362. [CrossRef]

3. Lundstrom, B.N.; Higgs, M.H.; Spain, W.J.; Fairhall, A.L. Fractional differentiation by neocortical pyramidal neurons. Nat. Neurosci. 2008, 11, 1335-1342. [CrossRef] [PubMed]

4. Magin, R.L.; Ovadia, M. Modeling the cardiac tissue electrode interface using fractional calculus. J. Vib. Control 2008, 14, 1431-1442. [CrossRef]

5. Picozzi, S.; West, B.J. Fractional Langevin model of memory in financial markets. Phys. Rev. E 2002, 66, 46-118. [CrossRef] [PubMed]

6. Rahmatullah; Ellahi, R.; Mohyud-Din, S.T.; Khan, U. Exact traveling wave solutions of fractional order Boussinesq-like equations by applying Exp-function method. Results Phys. 2018, 8, 114-120. [CrossRef]

7. Sohail, A.; Maqbool, K.; Ellahi, R. Stability analysis for fractional-order partial differential equations by means of space spectral time Adams-Bashforth Moulton method. Numer. Methods Partial. Differ. Equ. 2017, 34, 19-29. [CrossRef]

8. Tripathi, D.; Pandey, S.K.; Das, S. Peristaltic flow of viscoelastic fluid with fractional Maxwell model through a channel. Appl. Math. Comput. 2010, 215, 3645-3654. [CrossRef]

9. Duarte-Mermoud, M.A.; Aguila-Camacho, N.; Gallegos, J.A.; Castro-Linares, R. Using general quadratic Lyapunov functions to prove Lyapunov uniform stability for fractional order systems. Commun. Nonlinear Sci. Numer. Simul. 2015, 22, 650-659. [CrossRef]

10. Chen, L.P.; He, Y.G.; Chai; Y.; Wu, R.C. New results on stability stabilization of a class of nonlinear fractional-order systems. Nonlinear Dynam. 2014, 75, 633-641. [CrossRef]

11. Wu, G.C.; Abdeljawad, T.; Liu, J.; Baleanu, D.; Wu, K.T. Mittag-Leffler stability analysis of fractional discrete-time neural networks via fixed point technique. Nonlinear Anal. Model. Control 2019, 24, 919-936. [CrossRef]

12. Wu, G.C.; Baleanu, D.; Huang, L.L. Novel Mittag-Leffler stability of linear fractional delay difference equations with impulse. Appl. Math. Lett. 2018, 82, 71-78. [CrossRef]

13. Yang, X.; Li, C.; Huang, T. Mittag-Leffler stability analysis of nonlinear fractional-order systems with impulses. Appl. Math. Comput. 2017, 293, 416-422. [CrossRef]

14. Brzdek, J.; Eghbali, N. On approximate solutions of some delayed fractional differential equations. Appl. Math. Lett. 2016, 54, 31-35. [CrossRef]

15. Chen, L.P.; Liu, C.; Wu, R.C.; He, Y.G.; Chai, Y. Finite-time stability criteria for a class of fractional-order neural networks with delay. Neural Comput. Appl. 2016, 27, 549-556. [CrossRef]

16. Li, M.; Wang, J.R. Finite time stability of fractional delay differential equations. Appl. Math. Lett. 2017, 64, 170-176. [CrossRef]

17. Liu, S.; Li, X.; Zhou, X.F.; Jiang, W. Lyapunov stability analysis of fractional nonlinear systems. Appl. Math. Lett. 2016, 51, 13-19. [CrossRef]

18. Liu, S.; Zhou, X.F.; Li, X.; Jiang, W. Stability of fractional nonlinear singular systems its applications in synchronization of complex dynamical networks. Nonlinear Dynam. 2016, 84, 2377-2385. [CrossRef]

19. Ozarslan, M.A.; Ustaoglu, C. Some incomplete hypergeometric functions and incomplete Riemann-Liouville fractional integral operators. Mathematics 2019, 7, 483. [CrossRef] 
20. Rashid, S.; Abdeljawad, T.; Jarad, F.; Noor, M.A. Some estimates for generalized Riemann-Liouville fractional integrals of exponentially convex functions and their applications. Mathematics 2019, 7, 807. [CrossRef]

21. Fridman, E. Stability of linear descriptor systems with delays: a Lyapunov-based approach. Aust. J. Math. Anal. Appl. 2002, 273, 24-44. [CrossRef]

22. Kwon, O.M.; Park, J.H.; Lee, S.M. Augmented Lyapunov functional approach to stability of uncertain neutral systems with time-varying delays. Appl. Comput. Math. 2009, 207, 202-212. [CrossRef]

23. Liao, X.; Liu, Y.; Guo, S.; Mai, H. Asymptotic stability of delayed neural networks: a descriptor system approach. Commun. Nonlinear Sci. Numer. Simul. 2009, 14, 3120-3133. [CrossRef]

24. Park, J.H. Delay-dependent criterion for guaranteed cost control of neutral delay systems. J. Opt. Theory Appl. 2005, 124, 491-502. [CrossRef]

25. Deng, S.; Liao, X.; Guo, S. Asymptotic stability analysis of certain neutral differential equations: A descriptor system approach. Math. Comput. Simul. 2009, 71, 4297-4308. [CrossRef]

26. Kwon, O.M.; Park, J.H. On improved delay-dependent stability criterion of certain neutral differential equations. Appl. Math. Comput. 2008, 199, 385-391. [CrossRef]

27. Nam, P.T.; Phat, V.N. An improved stability criterion for a class of neutral differential equations. Appl. Math. Lett. 2009, 22, 31-35. [CrossRef]

28. Agarwal, R.P.; Grace, S.R. Asymptotic stability of certain neutral differential equations. Math. Comput. Model. 2000, 31, 9-15. [CrossRef]

29. Park, J.H.; Kwon, O.M. Stability analysis of certain nonlinear differential equation. Chaos Solitons Fractals 2008, 27, 450-453. [CrossRef]

30. Chen, H. Some improved criteria on exponential stability of neutral differential equation. Adv. Differ. Equ. 2012, 2012, 170. [CrossRef]

31. Chen, H.; Meng, X. An improved exponential stability criterion for a class of neutral delayed differential equations. Appl. Math. Lett. 2011, 24, 1763-1767. [CrossRef]

32. Keadnarmol, P.; Rojsiraphisal, T. Globally exponential stability of a certain neutral differential equation with time-varying delays. Adv. Differ. Equ. 2014, 2014, 32. [CrossRef]

33. Li, X. Global exponential stability for a class of neural networks. Appl. Math. Lett. 2009, 22, 1235-1239. [CrossRef]

34. Li, H.; Zhou, S.; Li, H. Asymptotic stability analysis of fractional-order neutral systems with time delay. Adv. Differ. Equ. 2015, 2015, 325-335. [CrossRef]

35. Liu, S.; Wu, X.; Zhang, Y.J.; Yang, R. Asymptotical stability of Riemann-Liouville fractional neutral systems. Appl. Math. Lett. 2017, 69, 168-173. [CrossRef]

36. Podlubny, I. Fractional Differential Equations; Academic Press: New York, NY, USA, 1999.

37. Kilbas, A.A.; Srivastava, H.M.; Trujillo, J.J. Theory and Application of Fractional Differential Equations; Elsevier: New York, NY, USA, 2006.

38. Rojsiraphisal, T.; Niamsup, P. Exponential stability of certain neutral differential equations. Appl. Math. Lett. 2010, 17, 3875-3880. [CrossRef]

(c) 2020 by the authors. Licensee MDPI, Basel, Switzerland. This article is an open access article distributed under the terms and conditions of the Creative Commons Attribution (CC BY) license (http://creativecommons.org/licenses/by/4.0/). 